\title{
A novel three pseudo-component approach (ThPCA) for thermodynamic description of hydrocarbon-water systems
}

\author{
Mehrak Mahmudi $\cdot$ Mohammad Taghi Sadeghi
}

Received: 27 April 2013/Accepted: 29 July 2013/Published online: 17 August 2013

(C) The Author(s) 2013. This article is published with open access at Springerlink.com

\begin{abstract}
Thermodynamic description of the hydrocarbon processes facing considerable variation in composition such as gas-lift process require an accurate and speedy algorithm to perform the three-phase multi-component flash calculation based on an equation of state. A novel approach for thermodynamic description of the three-phase fluid is introduced in this study to overcome the complex and time-consuming multi-component flash calculation procedure. The constituents of each pseudo-component and their properties were determined employing an optimization method minimizing the difference between the results of the three pseudo-component approach (ThPCA) with those from the compositional approach. Performance results both in accuracy and computing speed for the ThPCA method showed that using the three optimum pseudo-components with flash calculations based on an equation of state, reduce the computational time by half compared to the compositional model while it only decreases the accuracy by $5 \%$.
\end{abstract}

Keywords Thermodynamic description - Three pseudo-component approach · Compositional model .

Gas-lift process

\section{List of symbols \\ $f \quad$ Fugacity \\ $G \quad$ Vapor phase fraction}

M. Mahmudi · M. T. Sadeghi ( $\square)$

Process Simulation and Control Research Lab., Department

of Chemical Engineering, Iran University of Science and

Technology (IUST), Narmak, 16844 Tehran, Iran

e-mail: sadeghi@iust.ac.ir

M. Mahmudi

e-mail: mahmudi@iust.ac.ir
$K \quad$ Equilibrium constant

$P \quad$ Pressure (bar)

$x \quad$ Mole fraction of oil pseudo-component

$y \quad$ Mole fraction of gas pseudo-component

$z$ Feed composition

$O$ Hydrocarbon-rich liquid phase fraction

$w$ Mole fraction of water pseudo-component

$W$ Water-rich liquid phase fraction

$\varnothing \quad$ Fugacity coefficient

\section{Superscripts}

O Hydrocarbon-rich phase indicator

G Vapor phase indicator

W Water-rich phase indicator

\section{Subscripts}

COM Compositional approach

psg Gas pseudo-component

pso Oil pseudo-component

psw Water pseudo-component

ThPCA Three pseudo-component approach

\section{Introduction}

Hydrocarbon-water fluid systems consist of different components including hydrocarbons and water distributed in the three phases of water-rich liquid, hydrocarbon-rich liquid and vapor. Accuracy and speed of computation for modeling these systems depend directly on the complexity of the thermodynamic description of the involved fluids. Most studies such as Guanren (1986), Shi et al. (2005), Cazarez-Candia and Vasquez-Cruz (2005) as well as Hasan et al. (2007) performed the calculations for the hydrocarbon-water system based on the simplified black-oil approach. In the black-oil model, two pseudo-components

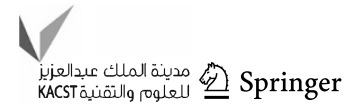


namely oil and gas are utilized to describe the hydrocarbon fluid system. A third pseudo-component is also employed describing the water. These pseudo-components are usually defined as distinct phases at standard conditions. For example, the gas (vapor) and oil (liquid) at surface conditions are labeled as the gas and oil pseudo-components, respectively. Here, only the gas component is allowed to dissolve in the oil phase, while fluid properties can be supplied as functions of pressure and temperature via data tables, or calculated from the built-in correlations.

The wise approach to predict the phase behavior is a compositional description in which all components are allowed to distribute between the phases. Compositional model deals with quite a large number of components using equilibrium flash calculations for all components as well as $K$ values and an equation of state to determine the phase compositions. In principle, a mass balance for each component is made in a compositional model. Development of compositional modeling with equation of state formulations has received an increasing attention in recent years (Varavei and Sepehrnoori 2009).

The price of more accurate compositional treatment is a huge increase in computational time. To limit the computation time, a common practice is to lump, or to pseudoize the fluid description. Thus, the pure compounds of the fluid are categorized into a number of component groups, termed as pseudo-components. It is obvious that the pseudoization will lead to losses in accuracy and flexibility in the equation of state calculations. Therefore, considerable effort has been put into the task of formulating pseudoization methods, in which the fluids can be described as accurately as possible, and as few pseudo-components as possible. All existing pseudoization procedures can be divided into two steps: the selection of pseudo-component groups and calculation of physical properties needed in the relevant EOS. For cubic EOS, these properties are the critical temperature, critical pressure, acentric factor and binary interaction coefficients. A number of different main categories for grouping methods exist. In the most simple method, components are grouped based on fixed rules, independent of the nature of the relevant fluid (Wu and Batycky 1988; Hong 1982). Other authors have chosen to form pseudocomponents which are approximately equal in size (Kay 1936; Pedersen et al. 1982). In some more theoretically based models, components with similar properties are grouped together (Lee et al. 1981; Montel and Gouel 1984; Danesh et al. 1990; Schlijper and van Bergen 1991; Lomeland and Harstad 1995). In the last category of grouping methods, component groups are defined on the basis of EOS calculations performed on the original unlumped fluid descriptions (Mehra and Heidmann 1982; Li et al. 1985; Newley and Merill 1991). Moreover, the property calculation methods can be divided into a number of different categories. In the most primitive form, pseudo-component properties are calculated on the basis of pure component properties employing different averaging techniques (Hong 1982; Kay 1936; Pedersen et al. 1982). In some more recent methods, the mixing rules of the EOS are utilized to calculate pseudo-component properties (Danesh et al. 1990; Lomeland and Harstad 1995; Mehra and Heidmann 1982; Li et al. 1985; Newley and Merill 1991; Leibovici 1993). Finally, a number of authors have used EOS calculations to adjust some parameters used in the property calculation methods (Wu and Batycky 1988; Li et al. 1985; Newley and Merill1991).

The objective of this work is to present a three pseudocomponent approach that can deliver accuracy similar to a compositional model at a much reduced computational cost. To do this, two existing lumping procedures are used (Hong and Leibovici methods). In this study, an optimal selection of pseudo-component groups, and calculation of physical properties are also presented. The proposed method is evaluated modeling a gas-lift process as a casestudy with two different fluids. In all the calculations, Peng-Robinson equation of state is used with the classical mixing rules.

\section{The three pseudo-component approach procedure}

A new thermodynamic description named "three pseudocomponent approach" (ThPCA) is proposed in this article. The main idea is to take advantage of the speed of black-oil model employing the pseudo-components scheme while keeping the accuracy of compositional model utilizing the flash calculations based on an equation of state.

In ThPCA, the hydrocarbon-water fluid is composed of three pseudo-components including the oil pseudo-component (suffix pso), gas pseudo-component (suffix psg) and water pseudo-component (suffix psw). The three pseudocomponents are distributed in the three phases of water liquid, hydrocarbon-rich liquid and vapor. Solubility of hydrocarbon components in the water liquid phase is neglected, implying the phase consists of pure water. This assumption is validated by Lapene et al. (2010) for three synthetic mixtures containing hydrocarbons and water for two reservoir fluid-water systems. The assumption results were in very good agreement with those from the full scale three-phase flash calculation.

Two points should be considered when grouping the constituents in pseudo-components not losing the accuracy of the equation of state prediction: the selection of each pseudo-component involved and the way to calculate their physical properties. Accordingly, the ThPCA procedure has three steps: (1) select the pseudo-component groups (2) calculate the physical properties needed in the relevant 
EOS including the critical temperatures, critical pressure, acentric factors and binary interaction coefficients and (3) perform the three-phase stability and flash calculations.

Selection of the three pseudo-components

The first step in application of ThPCA is the selection of each pseudo-component. In standard black-oil model, the pseudo-components are usually defined as phases at standard conditions. For example, the gas and oil at surface conditions are labeled as the gas and oil pseudo-components, respectively. The proposed method in this study is selecting the components lumped in each pseudo-component using optimization concept. To optimally select the pseudo-components, various grouped components in the oil pseudo-component are examined. The selected option should lead to minimum difference with the compositional model results. To do this, the following error function was defined that uses the values of phase fractions obtained at equilibrium conditions based on ThPCA and compositional model:

$$
\begin{aligned}
& \text { Error }=\sum_{T} \sum_{p}\left[\left(G_{\mathrm{COM}}-G_{\mathrm{ThSCA}}\right)^{2}+\left(O_{\mathrm{COM}}-O_{\mathrm{ThSCA}}\right)^{2}\right. \\
& \left.+\left(W_{\mathrm{COM}}-W_{\mathrm{ThSCA}}\right)^{2}\right]
\end{aligned}
$$

where $G, O$ and $W$ are the gas, oil and water phase fractions at each temperature and pressure calculated by flash calculations based on PR-EOS. The subscript ThPCA and COM reflects the three pseudo-component and compositional approaches. The above error function compares the results of ThPCA with a compositional model in a range of pressure and temperature values.

\section{Calculation of pseudo-component properties}

The next step after selecting the contents of each pseudocomponent is choosing the property calculation method. Pseudo-component properties can be calculated using present methods. In this study, Hong correlations (Hong 1982) that use averaging techniques and Leibovici correlations (Leibovici 1993) that utilize the mixing rules of the EOS to calculate pseudo-component properties are selected. Optimization concept was employed to calculate the pseudo-component properties including critical temperature, critical pressure, acentric factor and binary interaction coefficients. This scheme aims at minimizing the difference between the phase fraction values taken from the compositional system with those from the three pseudocomponent approach. It ensures that the predicted phase behavior using ThPCA is not altered significantly compared to the compositional approach.

Flash calculations of the three pseudo-components

The three pseudo-components distribution in the phases $O$, $G$ and $W$ is determined by phase equilibrium calculations. This requires molar-balance constraint to be preserved while chemical potentials of each component are the same for all phases, and the Gibbs free energy at constant temperature and pressure is minimized. In free water flash method (Lapene et al. 2010), these can be described for a mixture of hydrocarbon-water fluid having the three pseudo-components pso, psg and psw as:

$f_{\text {pso }}^{O}=f_{\text {pso }}^{G}$

$f_{\mathrm{psg}}^{O}=f_{\mathrm{psg}}^{G}$

$f_{\mathrm{psw}}^{O}=f_{\mathrm{psw}}^{G}=f_{\mathrm{psw}}^{W}$

and using fugacity coefficients as:

$P \varnothing_{\mathrm{pso}}^{O} x^{O}=P \varnothing_{\mathrm{pso}}^{G} x^{G}$

$P \varnothing_{\mathrm{psg}}^{O} y^{O}=P \varnothing_{\mathrm{psg}}^{G} y^{G}$

$P \varnothing_{\mathrm{psw}}^{O} w^{O}=P \varnothing_{\mathrm{psw}}^{G} w^{G}=P \varnothing_{\mathrm{psw}}^{W} w^{W}$

where $x, y, w$ are the mole fractions of oil, gas and water pseudo-components in each phase, respectively.

The equilibrium ratios are defined as:

$K_{\mathrm{pso}}^{I}=\frac{x^{G}}{x^{O}}=\frac{\varnothing_{\mathrm{pso}}^{O}}{\varnothing_{\mathrm{pso}}^{G}}$

$K_{\mathrm{psg}}^{I}=\frac{y^{G}}{y^{O}}=\frac{\varnothing_{\mathrm{psg}}^{O}}{\varnothing_{\mathrm{psg}}^{G}}$

$K_{\mathrm{psw}}^{I}=\frac{w^{G}}{w^{O}}=\frac{\varnothing_{\mathrm{psw}}^{O}}{\varnothing_{\mathrm{psw}}^{G}}$

$K_{\mathrm{psw}}^{I I}=\frac{w^{G}}{w^{W}}=\frac{\varnothing_{\mathrm{psw}}^{W}}{\varnothing_{\mathrm{psw}}^{G}}$

In a free-water system, water is distributed over the three phases, while the oil and gas pseudo-components are present only in gas and hydrocarbon (non-aqueous) phases. This assumption reads:

$w^{W}=1$.

Thus, Eq. (11) is simplified for the water component and takes the following form: 
$w^{G}=K_{\mathrm{psw}}^{I I}$

and from Eq. (10):

$w^{G}=\frac{K_{\mathrm{psw}}^{I I}}{K_{\mathrm{psw}}^{I}}$

The component material balances for gas/oil/water system are:

$z_{\text {pso }}=x^{O} O+x^{G} G$

$z_{\mathrm{psg}}=y^{O} O+y^{G} G$

$z_{\mathrm{psw}}=w^{O} O+w^{G} G+w^{W} W$

and the overall material balance equation is:

$G+O+W=1$

Using Eqs. (14)-(18) for the water component, an expression for the water phase fraction is obtained:

$W=\frac{z_{\mathrm{psw}}+G\left(w^{O}-w^{G}\right)-w^{O}}{1-w^{O}}$

For oil and gas pseudo-components, Eqs. (15 and 16) can be written using Eqs. (8-9 and 18) as follows:

$z_{\mathrm{pso}}=x^{O} K_{\mathrm{pso}}^{I} G+x^{O}(1-G-W)$

$z_{\mathrm{psg}}=y^{O} K_{\mathrm{psg}}^{I} G+y^{O}(1-G-W)$

Finally, using Eq. (19), the expressions for mole fractions of pseudo-components in hydrocarbon-rich liquid and vapor phases as functions of feed composition, equilibrium constants and vapor fraction are obtained from Eqs. (20 and 21):

$$
\begin{aligned}
x^{O} & =\frac{z_{\mathrm{pso}}}{1+G\left(K_{\mathrm{pso}}^{I}-1\right)-W} \\
& =\frac{z_{\mathrm{pso}}}{1+G\left(K_{\mathrm{pso}}^{I}-1+\frac{w^{G}-w^{O}}{1-w^{O}}\right)+\frac{w^{O}-z_{\mathrm{psw}}}{1-w^{O}}}
\end{aligned}
$$

$$
\begin{aligned}
y^{O} & =\frac{z_{i}}{1+V\left(K_{\mathrm{psg}}^{I}-1\right)-W} \\
& =\frac{z_{\mathrm{psg}}}{1+V\left(K_{\mathrm{psg}}^{I}-1+\frac{w^{G}-w^{O}}{1-w^{O}}\right)+\frac{w^{O}-z_{\mathrm{psw}}}{1-w^{O}}} \\
x^{G} & =x^{O} K_{\mathrm{pso}}^{I} \\
y^{G} & =y^{O} K_{\mathrm{psg}}^{I}
\end{aligned}
$$

\section{Results and discussion}

Test examples include two various three-phase equilibrium calculations for two different reservoir fluid-water systems. The Peng-Robinson cubic equation of state is used in the two case-studies. The results obtained from the new three pseudo-component approach are compared with those of compositional model.

Case-study 1

An illustrative example based on a mature Iranian oil field was used to evaluate the three pseudo-component approach. The hydrocarbon-water system is considered as $100 \mathrm{~mol}$ of hydrocarbon with fluid characteristics shown in Table 1 and $50 \mathrm{~mol}$ of water. The first step is to select the components that can be lumped in oil and gas pseudocomponents. Various options were considered for the hydrocarbon components in the oil pseudo-component. Next, the ThPCA flash calculation results were compared to the compositional flash results. The option that showed minimal differences, identify the hydrocarbon components that must be considered in each pseudo-component. Criti-

\begin{tabular}{|c|c|c|c|c|c|c|}
\hline No. & Component & Composition (mol) & $M_{\mathrm{w}}(\mathrm{g} / \mathrm{mole})$ & $T_{\mathrm{c}}(\mathrm{K})$ & $P_{\mathrm{c}}(\mathrm{bar})$ & $\omega$ \\
\hline 1 & $\mathrm{CO}_{2}$ & 1.63 & 44.01 & 304.21 & 73.77 & 0.2250 \\
\hline 2 & $\mathrm{H}_{2} \mathrm{~S}$ & 0.17 & 34.08 & 373.60 & 90.08 & 0.0810 \\
\hline 3 & $\mathrm{~N}_{2}$ & 0.25 & 28.01 & 126.20 & 33.94 & 0.0400 \\
\hline 4 & $\mathrm{C}_{1}$ & 14.39 & 16.04 & 190.60 & 46.00 & 0.0115 \\
\hline 5 & $\mathrm{C}_{2}$ & 7.13 & 30.07 & 305.40 & 48.84 & 0.0908 \\
\hline 6 & $\mathrm{C}_{3}$ & 8.08 & 44.10 & 369.80 & 42.46 & 0.1454 \\
\hline 7 & $i-\mathrm{C}_{4}$ & 1.67 & 58.12 & 408.10 & 36.48 & 0.1760 \\
\hline 8 & $n-\mathrm{C}_{4}$ & 4.67 & 58.12 & 425.20 & 38.00 & 0.1928 \\
\hline 9 & $i-\mathrm{C}_{5}$ & 1.61 & 71.94 & 464.74 & 34.77 & 0.2235 \\
\hline 10 & $\mathrm{n}-\mathrm{C}_{5}$ & 2.95 & 72.15 & 469.60 & 33.74 & 0.2273 \\
\hline 11 & $\mathrm{C}_{6}$ & 5.42 & 84.99 & 515.28 & 32.57 & 0.2637 \\
\hline 12 & $\mathrm{C}_{7}^{+}$ & 52.03 & 243.0 & 744.47 & 17.17 & 0.8561 \\
\hline
\end{tabular}
cal properties, acentric factors and the binary interaction coefficients are required for the flash calculations.

To calculate the pseudo-component properties, Hong (1982) and Leibovici (1993) correlations were utilized.

Table 1 Composition and component properties of the hydrocarbon fluid (case-study 1) 
Table 2 Optimum values of the three pseudo-component properties

\begin{tabular}{llccccccc}
\hline Pseudo-component & Composition & $M_{w}(\mathrm{~g} / \mathrm{mol})$ & $P_{c}(\mathrm{bar})$ & $T_{c}(\mathrm{~K})$ & $\omega$ & $k_{\mathrm{H} 2 \mathrm{O}, \mathrm{j}}$ & $K_{\mathrm{ps}-\mathrm{oil}, \mathrm{j}}$ & $K_{\mathrm{ps}-\mathrm{gas}, \mathrm{j}}$ \\
\hline Water & 0.35 & 18.02 & 221.20 & 647.37 & 0.344 & - & 0.5 \\
PS-oil & 0.45 & 228.69 & 20.15 & 581.71 & 0.350 & 0.5 & - \\
PS-gas & 0.2 & 33.19 & 36.45 & 228.04 & 0.151 & 0.5 & 0.00216 & - \\
\hline
\end{tabular}

Moreover, optimization concept was employed to calculate the pseudo-component properties including the critical temperatures, critical pressures, acentric factors and binary interaction coefficients. This scheme aims at minimizing the difference between phase fraction values of the compositional system with those from the three pseudo-component approach. This idea ensures that the predicted phase behavior using ThPCA is not altered significantly compared to the compositional approach. For this purpose, a computer program was developed, in which an error function was calculated after selection of pseudo-components, as Eq. (1).

This error function calculates the results of phase equilibrium calculations in a temperature range (in this study, between 305 and $360 \mathrm{~K}$ ) and a pressure range (in this study, 1-170 bar). The optimum values of pseudocomponent properties that minimized the above error function are shown in Table 2. Results of calculated phase fractions from both methods of ThPCA and compositional model for liquid hydrocarbon-rich, vapor and water are depicted in Fig. 1. In this figure, the results of ThPCA obtained using Hong (1982) and Leibovici (1993) correlations along with optimum property values are compared with those taken from compositional approach. The results show a good agreement between ThPCA and compositional flash outcome. Figure 1 and Table 3 show that the equilibrium results using Hong correlations further deviate from the compositional one compared to the results based on Leibovici correlations. This is because the Leibovici method is based on using the mixing rules of the EOS to
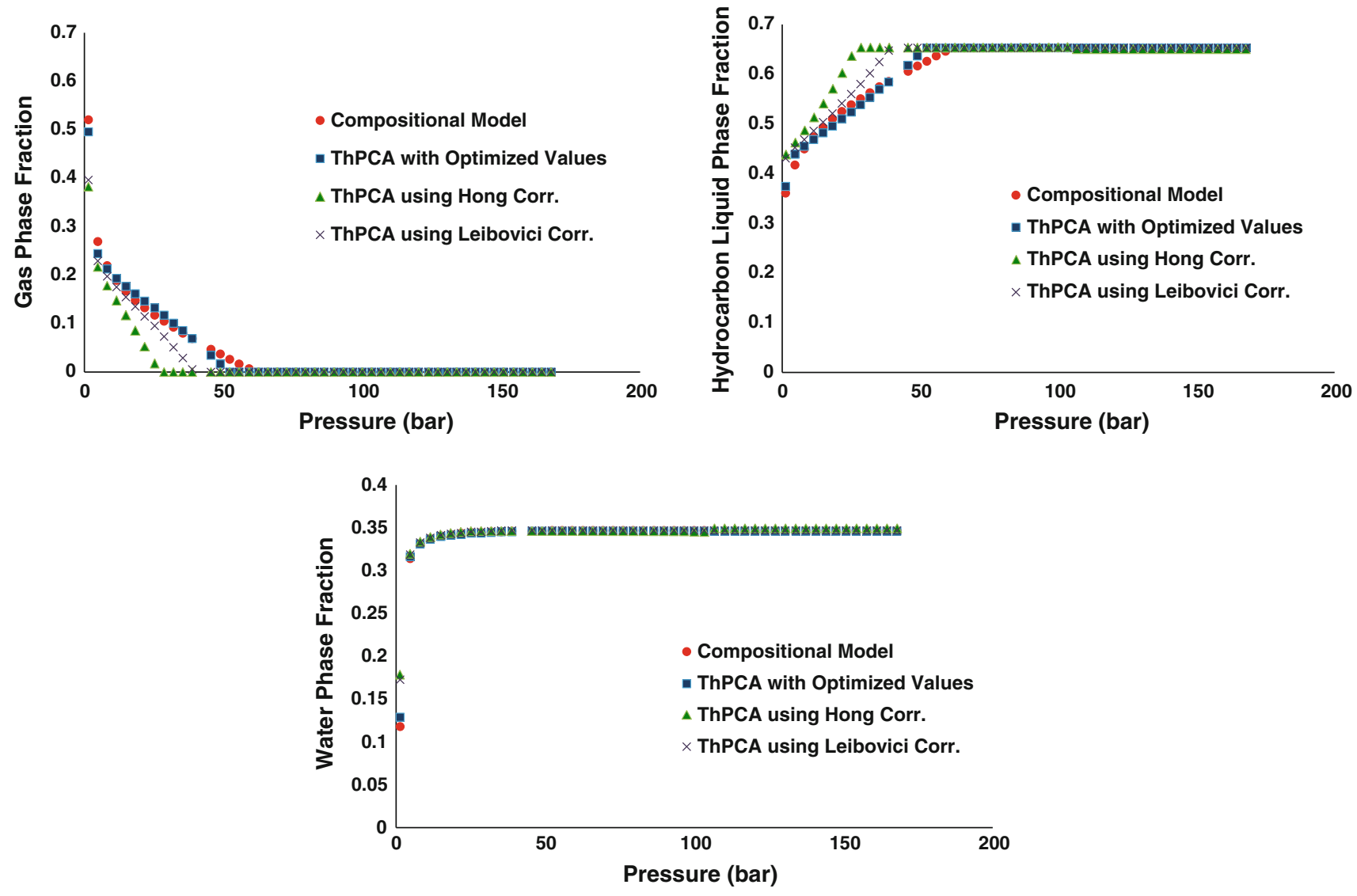

Fig. 1 Comparison between the three pseudo-component approach and compositional model 
Table 3 Comparison of the three pseudo-component approach and compositional model

\begin{tabular}{|c|c|c|c|c|c|c|c|c|c|c|c|c|}
\hline \multirow[t]{2}{*}{ Pressure (bar) } & \multicolumn{3}{|c|}{ Compositional model } & \multicolumn{3}{|c|}{ ThPCA with optimized values } & \multicolumn{3}{|c|}{ ThPCA using Hong corr. } & \multicolumn{3}{|c|}{ ThPCA using Leibovici Corr. } \\
\hline & $V$ & $O$ & $W$ & $V$ & $O$ & $W$ & $V$ & $O$ & $W$ & $V$ & $O$ & $W$ \\
\hline 1.36 & 0.5207 & 0.3609 & 0.1184 & 0.4961 & 0.3747 & 0.1292 & 0.3822 & 0.4386 & 0.1792 & 0.3959 & 0.4311 & 0.1730 \\
\hline 4.76 & 0.2688 & 0.4173 & 0.3139 & 0.2441 & 0.4393 & 0.3166 & 0.2169 & 0.4627 & 0.3203 & 0.2285 & 0.4527 & 0.3187 \\
\hline 8.16 & 0.2189 & 0.4496 & 0.3315 & 0.2129 & 0.4555 & 0.3317 & 0.1784 & 0.4871 & 0.3345 & 0.1976 & 0.4694 & 0.3329 \\
\hline 11.56 & 0.1888 & 0.4735 & 0.3377 & 0.1934 & 0.4693 & 0.3372 & 0.1470 & 0.5131 & 0.3399 & 0.1753 & 0.4864 & 0.3383 \\
\hline 14.97 & 0.1663 & 0.4929 & 0.3408 & 0.1770 & 0.4828 & 0.3401 & 0.1165 & 0.5408 & 0.3427 & 0.1550 & 0.5039 & 0.3412 \\
\hline 18.37 & 0.1478 & 0.5097 & 0.3426 & 0.1617 & 0.4964 & 0.3419 & 0.0851 & 0.5704 & 0.3445 & 0.1351 & 0.5220 & 0.3429 \\
\hline 21.77 & 0.1317 & 0.5246 & 0.3437 & 0.1467 & 0.5102 & 0.3431 & 0.0521 & 0.6021 & 0.3457 & 0.1150 & 0.5410 & 0.3441 \\
\hline 25.17 & 0.1173 & 0.5382 & 0.3445 & 0.1317 & 0.5244 & 0.3439 & 0.0171 & 0.6362 & 0.3466 & 0.0944 & 0.5607 & 0.3449 \\
\hline 28.57 & 0.1040 & 0.5509 & 0.3451 & 0.1165 & 0.5389 & 0.3446 & 0.0000 & 0.6530 & 0.3470 & 0.0732 & 0.5812 & 0.3456 \\
\hline 31.97 & 0.0916 & 0.5629 & 0.3455 & 0.1010 & 0.5539 & 0.3451 & 0.0000 & 0.6530 & 0.3470 & 0.0513 & 0.6026 & 0.3461 \\
\hline 35.37 & 0.0799 & 0.5743 & 0.3458 & 0.0852 & 0.5693 & 0.3455 & 0.0000 & 0.6530 & 0.3470 & 0.0285 & 0.6250 & 0.3465 \\
\hline 38.78 & 0.0686 & 0.5853 & 0.3461 & 0.0689 & 0.5853 & 0.3458 & 0.0000 & 0.6531 & 0.3469 & 0.0048 & 0.6484 & 0.3468 \\
\hline 42.18 & 0.0577 & 0.5960 & 0.3463 & 0.0521 & 0.6018 & 0.3461 & 0.0000 & 0.6531 & 0.3469 & 0.0000 & 0.6532 & 0.3468 \\
\hline 45.58 & 0.0471 & 0.6064 & 0.3465 & 0.0348 & 0.6189 & 0.3463 & 0.0000 & 0.6531 & 0.3469 & 0.0000 & 0.6532 & 0.3468 \\
\hline 48.98 & 0.0367 & 0.6167 & 0.3466 & 0.0169 & 0.6367 & 0.3465 & 0.0000 & 0.6531 & 0.3469 & 0.0000 & 0.6532 & 0.3468 \\
\hline 52.38 & 0.0265 & 0.6267 & 0.3467 & 0.0000 & 0.6534 & 0.3466 & 0.0000 & 0.6531 & 0.3469 & 0.0000 & 0.6532 & 0.3468 \\
\hline 55.78 & 0.0165 & 0.6367 & 0.3468 & 0.0000 & 0.6534 & 0.3466 & 0.0000 & 0.6531 & 0.3469 & 0.0000 & 0.6532 & 0.3468 \\
\hline 59.18 & 0.0066 & 0.6465 & 0.3469 & 0.0000 & 0.6534 & 0.3466 & 0.0000 & 0.6531 & 0.3469 & 0.0000 & 0.6532 & 0.3468 \\
\hline 62.59 & 0.0000 & 0.6530 & 0.3470 & 0.0000 & 0.6534 & 0.3466 & 0.0000 & 0.6532 & 0.3468 & 0.0000 & 0.6532 & 0.3468 \\
\hline 65.99 & 0.0000 & 0.6530 & 0.3470 & 0.0000 & 0.6534 & 0.3466 & 0.0000 & 0.6532 & 0.3468 & 0.0000 & 0.6532 & 0.3468 \\
\hline 69.39 & 0.0000 & 0.6530 & 0.3470 & 0.0000 & 0.6534 & 0.3466 & 0.0000 & 0.6532 & 0.3468 & 0.0000 & 0.6532 & 0.3468 \\
\hline 72.79 & 0.0000 & 0.6530 & 0.3470 & 0.0000 & 0.6534 & 0.3466 & 0.0000 & 0.6532 & 0.3468 & 0.0000 & 0.6532 & 0.3468 \\
\hline 76.19 & 0.0000 & 0.6530 & 0.3470 & 0.0000 & 0.6534 & 0.3466 & 0.0000 & 0.6532 & 0.3468 & 0.0000 & 0.6532 & 0.3468 \\
\hline 79.59 & 0.0000 & 0.6530 & 0.3470 & 0.0000 & 0.6534 & 0.3466 & 0.0000 & 0.6533 & 0.3467 & 0.0000 & 0.6532 & 0.3468 \\
\hline 82.99 & 0.0000 & 0.6530 & 0.3470 & 0.0000 & 0.6534 & 0.3466 & 0.0000 & 0.6533 & 0.3467 & 0.0000 & 0.6532 & 0.3468 \\
\hline 86.39 & 0.0000 & 0.6530 & 0.3470 & 0.0000 & 0.6534 & 0.3466 & 0.0000 & 0.6533 & 0.3467 & 0.0000 & 0.6532 & 0.3468 \\
\hline 89.80 & 0.0000 & 0.6530 & 0.3470 & 0.0000 & 0.6534 & 0.3466 & 0.0000 & 0.6534 & 0.3466 & 0.0000 & 0.6532 & 0.3468 \\
\hline 93.20 & 0.0000 & 0.6530 & 0.3470 & 0.0000 & 0.6534 & 0.3466 & 0.0000 & 0.6534 & 0.3466 & 0.0000 & 0.6532 & 0.3468 \\
\hline 96.60 & 0.0000 & 0.6530 & 0.3470 & 0.0000 & 0.6534 & 0.3466 & 0.0000 & 0.6535 & 0.3465 & 0.0000 & 0.6532 & 0.3468 \\
\hline 100.00 & 0.0000 & 0.6530 & 0.3470 & 0.0000 & 0.6534 & 0.3466 & 0.0000 & 0.6540 & 0.3460 & 0.0000 & 0.6532 & 0.3468 \\
\hline 103.40 & 0.0000 & 0.6530 & 0.3470 & 0.0000 & 0.6534 & 0.3466 & 0.0000 & 0.6542 & 0.3458 & 0.0000 & 0.6532 & 0.3468 \\
\hline 106.80 & 0.0000 & 0.6530 & 0.3470 & 0.0000 & 0.6534 & 0.3466 & 0.0000 & 0.6500 & 0.3500 & 0.0000 & 0.6532 & 0.3468 \\
\hline 110.20 & 0.0000 & 0.6530 & 0.3470 & 0.0000 & 0.6534 & 0.3466 & 0.0000 & 0.6500 & 0.3500 & 0.0000 & 0.6532 & 0.3468 \\
\hline 113.61 & 0.0000 & 0.6530 & 0.3470 & 0.0000 & 0.6534 & 0.3466 & 0.0000 & 0.6500 & 0.3500 & 0.0000 & 0.6533 & 0.3467 \\
\hline 117.01 & 0.0000 & 0.6530 & 0.3470 & 0.0000 & 0.6534 & 0.3466 & 0.0000 & 0.6500 & 0.3500 & 0.0000 & 0.6533 & 0.3467 \\
\hline 120.41 & 0.0000 & 0.6530 & 0.3470 & 0.0000 & 0.6534 & 0.3466 & 0.0000 & 0.6500 & 0.3500 & 0.0000 & 0.6533 & 0.3467 \\
\hline 123.81 & 0.0000 & 0.6530 & 0.3470 & 0.0000 & 0.6534 & 0.3466 & 0.0000 & 0.6500 & 0.3500 & 0.0000 & 0.6533 & 0.3467 \\
\hline 127.21 & 0.0000 & 0.6530 & 0.3470 & 0.0000 & 0.6534 & 0.3466 & 0.0000 & 0.6500 & 0.3500 & 0.0000 & 0.6533 & 0.3467 \\
\hline 130.61 & 0.0000 & 0.6530 & 0.3470 & 0.0000 & 0.6534 & 0.3466 & 0.0000 & 0.6500 & 0.3500 & 0.0000 & 0.6533 & 0.3467 \\
\hline 134.01 & 0.0000 & 0.6530 & 0.3470 & 0.0000 & 0.6534 & 0.3466 & 0.0000 & 0.6500 & 0.3500 & 0.0000 & 0.6533 & 0.3467 \\
\hline 137.41 & 0.0000 & 0.6530 & 0.3470 & 0.0000 & 0.6534 & 0.3466 & 0.0000 & 0.6500 & 0.3500 & 0.0000 & 0.6534 & 0.3466 \\
\hline 140.82 & 0.0000 & 0.6530 & 0.3470 & 0.0000 & 0.6534 & 0.3466 & 0.0000 & 0.6500 & 0.3500 & 0.0000 & 0.6534 & 0.3466 \\
\hline 144.22 & 0.0000 & 0.6530 & 0.3470 & 0.0000 & 0.6534 & 0.3466 & 0.0000 & 0.6500 & 0.3500 & 0.0000 & 0.6535 & 0.3465 \\
\hline 147.62 & 0.0000 & 0.6530 & 0.3470 & 0.0000 & 0.6534 & 0.3466 & 0.0000 & 0.6500 & 0.3500 & 0.0000 & 0.6535 & 0.3465 \\
\hline 151.02 & 0.0000 & 0.6530 & 0.3470 & 0.0000 & 0.6534 & 0.3466 & 0.0000 & 0.6500 & 0.3500 & 0.0000 & 0.6535 & 0.3465 \\
\hline 154.42 & 0.0000 & 0.6530 & 0.3470 & 0.0000 & 0.6535 & 0.3465 & 0.0000 & 0.6500 & 0.3500 & 0.0000 & 0.6534 & 0.3466 \\
\hline 157.82 & 0.0000 & 0.6530 & 0.3470 & 0.0000 & 0.6535 & 0.3465 & 0.0000 & 0.6500 & 0.3500 & 0.0000 & 0.6534 & 0.3466 \\
\hline
\end{tabular}


Table 3 continued

\begin{tabular}{|c|c|c|c|c|c|c|c|c|c|c|c|c|}
\hline \multirow[t]{2}{*}{ Pressure (bar) } & \multicolumn{3}{|c|}{ Compositional model } & \multicolumn{3}{|c|}{ ThPCA with optimized values } & \multicolumn{3}{|c|}{ ThPCA using Hong corr. } & \multicolumn{3}{|c|}{ ThPCA using Leibovici Corr. } \\
\hline & $V$ & $O$ & $W$ & $V$ & $O$ & $W$ & $V$ & $O$ & $W$ & $V$ & $O$ & $W$ \\
\hline 161.22 & 0.0000 & 0.6530 & 0.3470 & 0.0000 & 0.6535 & 0.3465 & 0.0000 & 0.6500 & 0.3500 & 0.0000 & 0.6534 & 0.3466 \\
\hline 164.63 & 0.0000 & 0.6530 & 0.3470 & 0.0000 & 0.6534 & 0.3466 & 0.0000 & 0.6500 & 0.3500 & 0.0000 & 0.6534 & 0.3466 \\
\hline 168.03 & 0.0000 & 0.6530 & 0.3470 & 0.0000 & 0.6534 & 0.3466 & 0.0000 & 0.6500 & 0.3500 & 0.0000 & 0.6534 & 0.3466 \\
\hline
\end{tabular}

Phase fraction, $V$ vapor phase, $O$ hydrocarbon-rich phase, $W$ water phase

determine the pseudo-component properties, while Hong method calculates them only using simple average relations.

Figure 2 shows the values of the liquid and vapor hydrocarbon-rich fractions obtained by ThPCA compared to the values obtained from compositional approach at different oil pseudo-component. As shown in the figure, if $C_{7}^{+}, \mathrm{C}_{6}, i \mathrm{C}_{5}, n \mathrm{C}_{5}, i \mathrm{C}_{4}$ and $C_{4}$ are lumped in one oil pseudocomponent and other components, except water are grouped in the gas pseudo-component, the ThPCA gives the nearest behavior to the compositional approach.

To investigate the speed and accuracy of ThPCA for pressure drop calculations of a well, the ThPCA calculations were applied to a gas-lifted well. Since the oil and gas compositions in a gas-lifted well are varied considerably, this may be a good case-study to evaluate the ThPCA. The particular
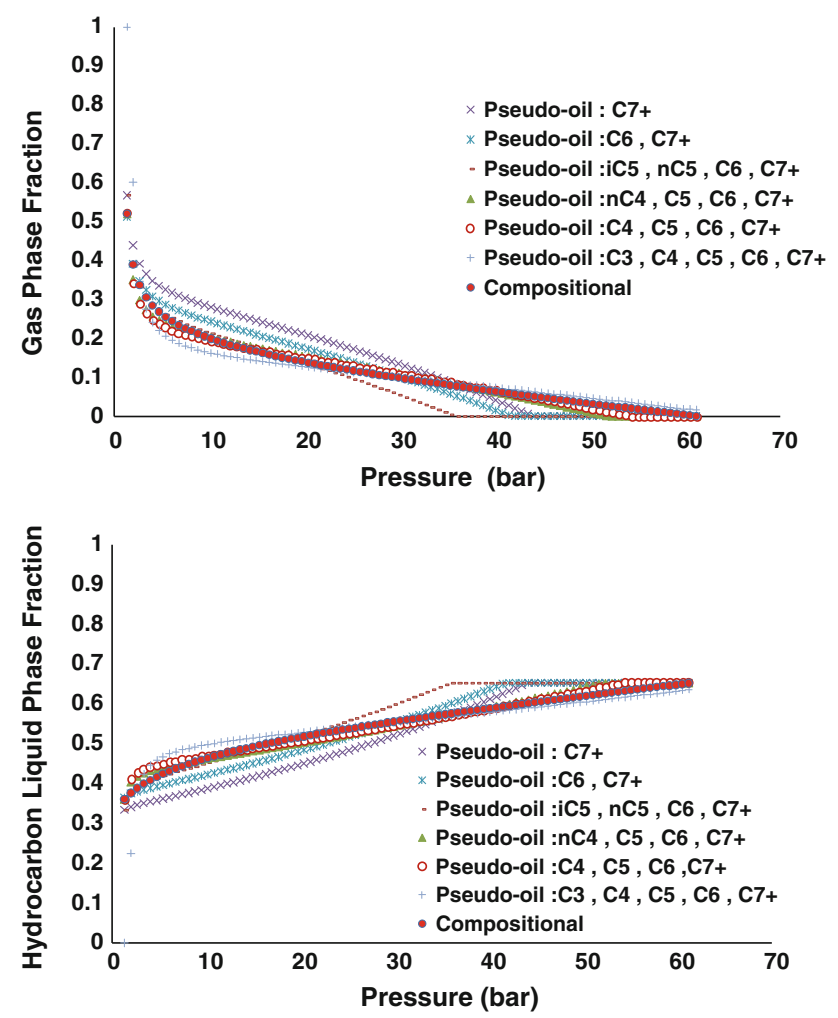

Fig. 2 Comparison between the three pseudo-component approach and compositional model well considered in this study had a tubing diameter of $0.127 \mathrm{~m}$ and a depth of 2,100 m equipped with a gas lift valve at $100 \mathrm{~m}$ above the sand face. The pressure at the bottom-hole is 172 bar. Pressure drop calculations for determining the wellhead pressure were performed. Figure 3 compares the results of calculated wellhead pressure as well as computational time at different gas injection rates at water-cut value of $20 \%$. The average relative error between the calculated wellhead pressure using ThPCA and compositional model is $3.6 \%$, while ThPCA reduces the computation time by $50 \%$.

According to the results, if the selection of components in each pseudo-component is properly chosen and their critical properties, acentric factors and binary interaction coefficients are correctly calculated, the phase equilibrium results will be in good agreement comparing ThPCA and compositional approaches.
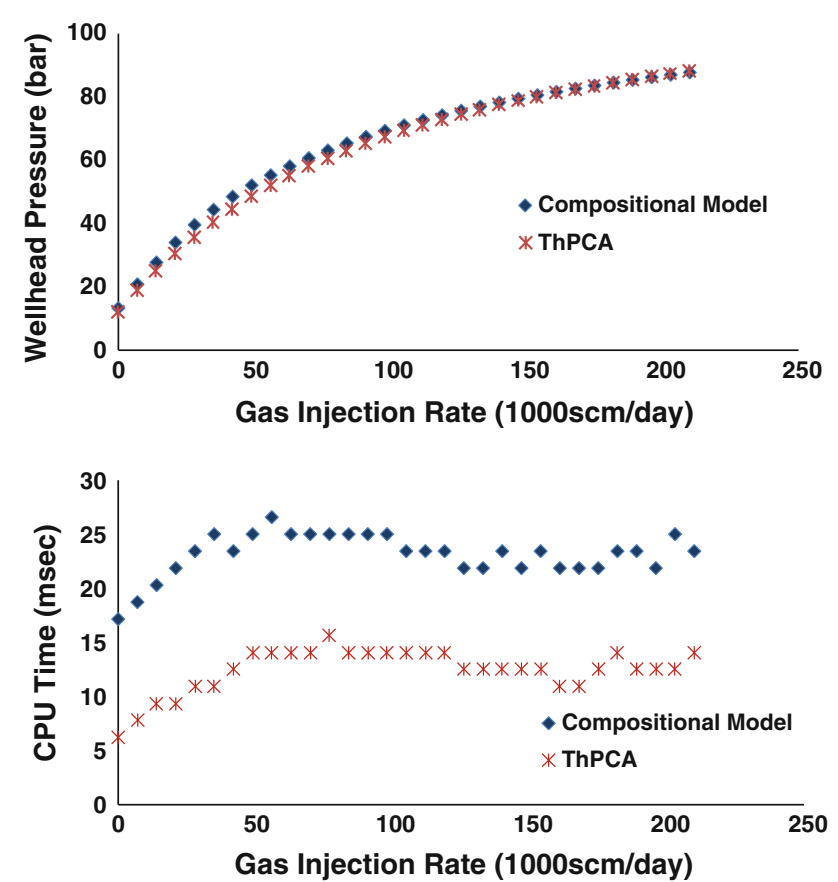

Fig. 3 Calculated wellhead pressure and computational time based on compositional model and ThPCA at different gas injection rates 
Table 4 Composition and component properties of the hydrocarbon fluid (case-study 2)

\begin{tabular}{|c|c|c|c|c|c|c|}
\hline No. & Component & Composition (mol) & $M_{\mathrm{w}}(\mathrm{g} / \mathrm{mol})$ & $T_{\mathrm{c}}(\mathrm{K})$ & $P_{\mathrm{c}}(\mathrm{bar})$ & $\omega$ \\
\hline 1 & $\mathrm{H}_{2} \mathrm{O}$ & 20 & 18.02 & 647.37 & 221.20 & 0.344 \\
\hline 2 & $\mathrm{~N}_{2}$ & 0.25 & 28.01 & 126.20 & 33.94 & 0.0400 \\
\hline 3 & $\mathrm{CO}_{2}$ & 1.63 & 44.01 & 304.21 & 73.77 & 0.2250 \\
\hline 4 & $\mathrm{C}_{1}$ & 14.39 & 16.04 & 190.60 & 46.00 & 0.0115 \\
\hline 5 & $\mathrm{C}_{2}$ & 7.13 & 30.07 & 305.40 & 48.84 & 0.0908 \\
\hline 6 & $\mathrm{C}_{3}$ & 8.08 & 44.10 & 369.80 & 42.46 & 0.1454 \\
\hline 7 & $i-\mathrm{C}_{4}$ & 1.67 & 58.12 & 408.10 & 36.48 & 0.1760 \\
\hline 8 & $n-\mathrm{C}_{4}$ & 4.67 & 58.12 & 425.20 & 38.00 & 0.1928 \\
\hline 9 & $i-\mathrm{C}_{5}$ & 1.61 & 71.94 & 464.74 & 34.77 & 0.2235 \\
\hline 10 & $n-\mathrm{C}_{5}$ & 2.95 & 72.15 & 469.60 & 33.74 & 0.2273 \\
\hline 11 & $\mathrm{C}_{6}$ & 5.42 & 84.99 & 515.28 & 32.57 & 0.2637 \\
\hline 12 & $\mathrm{C}_{7}$ & 1.15 & 97.87 & 553.84 & 31.00 & 0.2897 \\
\hline 13 & $\mathrm{C}_{8}$ & 1.07 & 111.54 & 581.28 & 28.50 & 0.3245 \\
\hline 14 & $\mathrm{C}_{9}$ & 0.95 & 126.10 & 609.35 & 26.50 & 0.3791 \\
\hline 15 & $\mathrm{C}_{10}$ & 0.67 & 140.14 & 626.97 & 24.60 & 0.4363 \\
\hline 16 & HVY1 & 1.65 & 179.30 & 658.15 & 21.20 & 0.5200 \\
\hline 17 & HVY1 & 1.13 & 290.60 & 778.15 & 15.70 & 0.6500 \\
\hline 18 & HVY1 & 0.22 & 450.00 & 998.15 & 13.50 & 0.7200 \\
\hline
\end{tabular}

Table 5 Optimum values of three pseudo-component properties

\begin{tabular}{llccccccc}
\hline Pseudo-component & Composition & $M_{w}(\mathrm{~g} / \mathrm{mol})$ & $P_{c}($ bar $)$ & $T_{c}(K)$ & $\omega$ & $k_{H 2 O, j}$ & $K_{p s-o i l, j}$ & $K_{p s-\text { gas }, j}$ \\
\hline Water & 0.17 & 18.02 & 221.20 & 647.37 & 0.3440 & - & 0.5 & 0.5 \\
PS-oil & 0.09 & 183.44 & 36.78 & 608.99 & 0.464 & 0.5 & - \\
PS-gas & 0.74 & 22.91 & 44.31 & 218.53 & 0.123 & 0.5 & 0.0063 & - \\
\hline
\end{tabular}

\section{Case-study 2}

The new three pseudo-component approach was also used to calculate the three-phase equilibrium in a gaseous hydrocarbon fluid. The composition (in moles) of a real reservoir fluid described by 18 components (Nichita et al. 2008) and component properties are given in Table 4. The results of optimum values of pseudo-component properties are listed in Table 5. The optimization results show that selection of $\mathrm{N}_{2}, \mathrm{CO}_{2}, C_{1}, C_{2}$ and $C_{3}$ in the gas pseudocomponents and others (except water) in oil pseudo-component leads to minimum deviation from the compositional results.

Speed and accuracy of the ThPCA in calculations of pressure drop for a well were studied. ThPCA calculations of the fluid were applied to the previous case well having $0.127 \mathrm{~m}$ of tubing diameter and depth of 2,100 m. Pressure drop calculations for determining the wellhead pressure at different values of water-cut were performed. Results of calculated wellhead pressure and computational time at different water-cut values are compared in Fig. 4. In this case-study, the average relative error between the calculated wellhead pressure using ThPCA and compositional is $2.5 \%$, while the computation time of ThPCA is about one-third of the compositional model.

The results of the above two studies show that the three pseudo-component approach introduced in this study reduces the computation time to less than half of the compositional approach with only $5 \%$ reduced accuracy. Even though, having higher number of components in the hydrocarbon fluid, the accuracy of the three pseudo-component approach results in greater savings in computational time. In using ThPCA to describe the thermodynamic behavior of a hydrocarbon-water fluid, it is important to properly select the components and secondly calculate the pseudo-component properties by minimizing the difference between results of ThPCA and those of compositional model.

\section{Conclusion}

Due to the high-speed of computing, the standard black-oil model is still used in industry for modeling and 

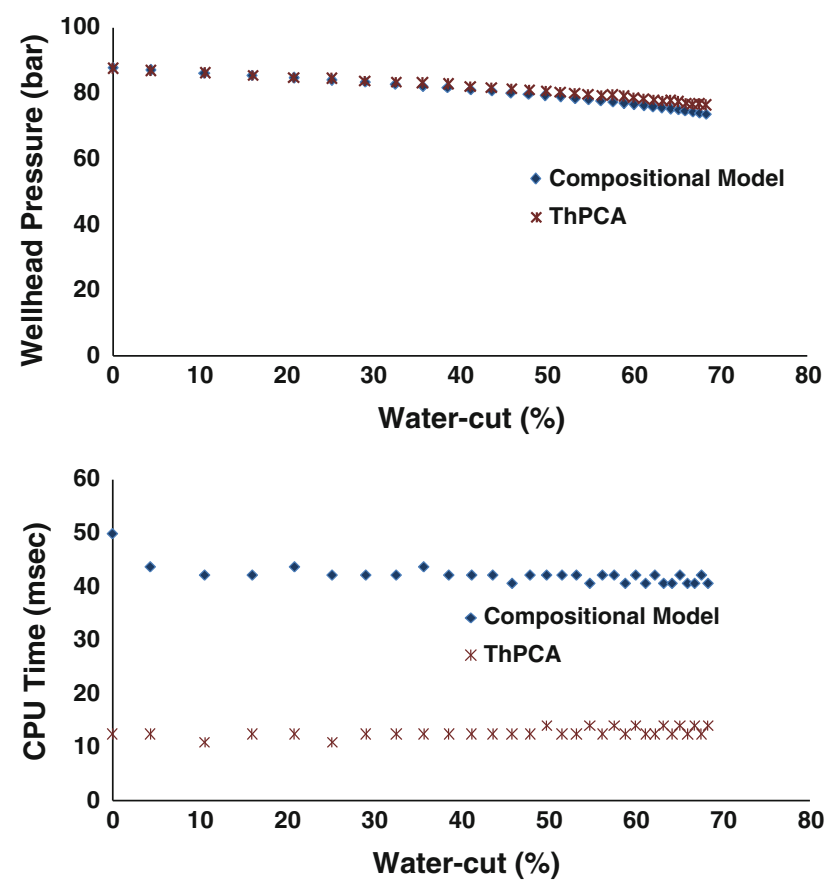

Fig. 4 Calculated wellhead pressure and computational time based on compositional model and ThPCA at different water-cut values

optimization of hydrocarbon processes. However, a simple black-oil model ignores the composition changes leading to significant error. In this study, a new approach called three pseudo-component approach was introduced to describe the three-phase hydrocarbon-water system. All components except water are lumped in two oil and gas pseudo-components in this approach. The constituents of each pseudocomponent and their properties were determined using optimization methods such that the difference between the results of thermodynamic calculations using ThPCA and compositional approach is minimal. The performance results of computing accuracy and speed using the new ThPCA showed that using three optimum pseudo-components along with flash calculations based on an equation of state, it reduces the computation time to less than half in the cost of $5 \%$ decreased accuracy. The ThPCA framework is applicable in modeling a wide range of practical fluid systems where the hydrocarbon-water mixture can be represented by three pseudo-components.

Open Access This article is distributed under the terms of the Creative Commons Attribution License which permits any use, distribution, and reproduction in any medium, provided the original author(s) and the source are credited.

\section{References}

Cazarez-Candia O, Vasquez-Cruz MA (2005) Prediction of pressure, temperature, and velocity distribution of two-phase flow in oil wells. J Pet Sci Eng 46:195-208
Danesh A, Xu D, Todd AC (1990) A grouping method to optimize oil description for compositional simulation of gas injection processes. In: Paper SPE 20745 presented at the 1990 SPE Annual Technical Conference and Exhibition. New Orleans, 23-26 September 1990

Guanren H (1986) The black oil model for a heavy oil reservoir. In: Paper SPE 14853 presented at the SPE International Meeting on Petroleum Engineering. Beijing, China, 17-20 March 1986

Hasan AR, Kabir CS, Sayarpour M (2007) A basic approach to wellbore two-phase flow modeling. In: Paper SPE 109868 presented at the 2007 SPE Annual Technical Conference and Exhibition. Anaheim. California, USA, 11-14 November 2007

Hong KC (1982) Lumped-component characterization of crude oils for compositional simulation. In: SPE/DOE Paper 10691 presented at the 3rd Joint Symposium on EOR. Tulsa, OK 4-7 April 1982

Kay WB (1936) Density of hydrocarbon gases and vapors. Ind Eng Chem 28(9):1014-1019

Lapene A, Nichita DV, Debenest G, Quintard M (2010) Three-phase free-water flash calculations using a new modified Rachford-rice equation. Fluid Phase Equ 297:121-128

Lee ST, Jacoby RH, Chen WH, Culham WE (1981) Experimental and theoretical studies on the fluid properties required for simulation of thermal processes. SPE J 21(5):535-550

Leibovici CF (1993) A consistent procedure for the estimation of properties associated to lumped systems. Fluid Phase Equ 87:189-197

Li YK, Nghiem LX, Siu A (1985) Phase behavior computations for reservoir fluids: effect of pseudo-components on phase diagrams and simulation results. J Can Pet Tech 24(6):29-37

Lomeland F, Harstad O (1995) Simplifying the task of grouping fluid components in compositional reservoir simulation. SPE Comp App 7(2):38-43

Mehra RK, Heidmann RA (1982) A statistical approach for combining reservoir fluids into pseudo components for compositional model studies. In: SPE paper 11201 presented at the 1982 Annual Technical Conference and Exhibition. New Orleans, LA, September 1982

Montel F, Gouel P (1984) A new lumping scheme of analytical data for composition studies. In: SPE Paper 13119 presented at the SPE 59th Annual Technical Conference. Houston, 16-19 September 1984

Newley TMJ, Merill RC Jr (1991) Pseudocomponent selection for compositional simulation. SPE Res Eng 6(4):490-496

Nichita DV, Broseta D, Elhorga P, Montel F (2008) Pseudocomponent delumping for multiphase equilibrium in hydrocarbon-water mixtures. Pet Sci Tech 26:2058-2077

Pedersen K, Thomassen P, Fredenslund A (1982) Phase equilibria and separation processes. Report SEP 8207. Institute for Kemiteknik, Denmark Tekniske Hojskole, Denmark

Schlijper AG, van Bergen ARD (1991) A free energy criterion for the selection of pseudocomponents for vapour/liquid equilibrium calculations. In: Astarita G, Sandler SI (eds) Kinetic and thermodynamic lumping of multicomponent mixtures. Elsevier, Amsterdam

Shi H, Holmes JA, Diaz LR, Durlofsky LJ, Aziz K (2005) Drift-flux parameters for three-phase steady-state flow in wellbores. In: Paper SPE 89836 presented at the SPE Annual Technical Conference and Exhibition. Houston, Texas, USA 26-29 September 2005

Varavei A, Sepehrnoori K (2009) An EOS-based compositional thermal reservoir simulator. In: Paper SPE 119154 presented in SPE Reservoir Simulation Symposium, The Wood-lands, Texas, 2-4 February 2009

Wu RS, Batycky JP (1988) Pseudocomponent component characterization for hydrocarbon miscible displacement. SPE Res Eng 3(3):875-883

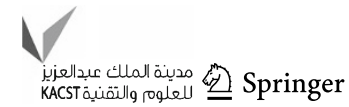

